\title{
Wie unterstützen Eltern die häuslichen Lernaktivitäten von Kindern mit Lernstörung?
}

\author{
Eine Längsschnittstudie
}

\author{
Janin Brandenburg ${ }^{1,2}$ und Sina S. Huschka ${ }^{1,2}$ \\ 'DIPF | Leibniz-Institut für Bildungsforschung und Bildungsinformation \\ ${ }^{2}$ Center for Research on Individual Development and Adaptive Education of Children at Risk (IDeA)
}

\begin{abstract}
Zusammenfassung: Theoretischer Hintergrund: Für Kinder mit Lernstörung (LS) hören die Lernproblemen nicht mit dem Unterrichtsschluss auf, sondern setzen sich bei den Hausaufgaben fort. Dennoch ist über die Hausaufgabenpraxis bei LS wenig bekannt. Fragestellung: Ziel war es, die familiäre Hausaufgabenpraxis bei Kindern mit und ohne LS längsschnittlich zu untersuchen. Methode: 82 Eltern (davon 47 von einem Kind mit LS) wurden zu vier Zeitpunkten befragt und latente Veränderungsmodelle gerechnet. Ergebnisse: Eltern von Kindern mit LS berichteten mehr Kontrolle und Konflikte bei den Hausaufgaben und nahmen ihre Hilfe als weniger kompetent wahr. Auch forderten sie von ihren Kindern weniger Anstrengung ein. Längsschnittlich nahmen bei beiden Gruppen die Hausaufgabenkontrolle sowie die familiäre Belastung ab. Diskussion und Schlussfolgerung: Die schwierigere Hausaufgabensituation bei Kindern mit LS legt nahe, das Thema bei der Diagnostik anzusprechen und zu eruieren, wie die Familien entlastet werden können.
\end{abstract}

Schlüsselwörter: elterliche Lernunterstützung, Hausaufgaben, Lernstörung, Längsschnittstudie

How Do Parents Support Homework Behavior in Children With Learning Disorders? A Longitudinal Study

Abstract: Theoretical Background: Although research suggests that parents become more involved in the homework process when their child exhibits problems in school, most research has not focused on children with learning disorders (LDs). Therefore, little is known about how parents of children with LDs support their children's learning. In addition, only a few longitudinal studies exist, even though parental homework involvement is likely to change throughout schooling. Objective: Parental homework involvement for children with and without LDs during the transition from primary to secondary school was examined. Specifically, we investigated (a) interindividual differences in the amount of homework support provided by parents of children with and without LDs, as well as (b) the linear trend and the interindividual stability of parents' homework involvement. Method: In this 2-year longitudinal study, 82 parents (47 parents of a child with LDs) completed a questionnaire on homework involvement at four measurement points every 6 months. The questionnaire consisted of four scales: (a) parental competence regarding help with homework, (b) parental homework control, (c) parent-child conflicts during homework, and (d) parents' effort attribution. Results: Bivariate correlations between the scales were mostly insignificant with two exceptions: (1) the more competent parents felt in helping with homework, the more they made use of control, and (2) parents who made more use of homework control, reported more parent-child conflicts than parents who controlled their child's homework to a lesser extent. Latent change score models revealed that parents continuously reduced homework control over the 2-year period and that parent-child conflicts during homework also significantly decreased - both for families of children with LDs and those without. The interindividual differences between parents, however, remained relatively constant over time, suggesting high rank-order stability of parental homework involvement. The results further showed that compared with parents of children without LDs, parents of children with LDs reported a higher frequency of homework control and increased homework conflict. They also felt less competent to effectively help with homework. Significant group differences were also found concerning parents' emphasis on effort: Parents of children with LDs attributed school success less strongly to effort. Discussion and Conclusion: Overall, the study showed that the homework situation is more stressful in families of children with LDs than in those without LDs. The longitudinal results further support the idea that parental homework involvement does not remain consistent over time but rather undergoes some changes as children grow older and transfer to secondary school. Thus, caution is warranted when generalizing results from cross-sectional studies on parental homework involvement to different grade levels.

Keywords: parental involvement, homework, learning disorder, longitudinal study

Lernstörungen gehören zu den häufigsten Entwicklungsstörungen in der Kindheit und sind ein Risikofaktor für
Schulversagen (Esser, Wyschkon \& Schmidt, 2002). Als Ursache gelten genetische Faktoren, die zu Problemen in 
den Bereichen der (neuro-)kognitive Informationsverarbeitung führen, die für den Erwerb von Schriftsprache und Mathematik relevant sind (Lonnemann, Linkersdörfer, Hasselhorn \& Lindberg, 2011; Schulte-Körne, Warnke \& Remschmidt, 2006). Umweltbedingungen können den Schweregrad der Lernstörungen zusätzlich beeinflussen. So können negative Rückmeldungen oder ungünstige Unterstützungsstrategien der Eltern und Lehrkräfte negativ auf das kindliche (Lern-)verhalten einwirken und dadurch die Lernprobleme verfestigen (Betz \& Breuninger, 1998). Umgekehrt können sich positive Umweltfaktoren wie eine gute häusliche Lernumwelt protektiv auswirken und eine genetische Prädisposition abmildern (Esmaeeli, Kyle \& Lundetræoder, 2019).

Daher kommt der Frage, wie Lernstörungen von den Familien bewältigt werden und in welcher Form die Eltern ihre Kinder schulisch unterstützen, eine zentrale Rolle zu. Denn das Auseinandersetzen mit den Lernproblemen hört für die Kinder nicht mit dem Unterrichtsschluss auf, sondern setzt sich auch in der Familie beim Erledigen der Hausaufgaben fort: Kinder mit Lernstörungen (LS) erleben mehr Probleme bei den Hausaufgaben und benötigen deutlich mehr Zeit als Kinder ohne LS (Bryan, Burstein \& Bryan, 2001). Auch berichten Eltern, dass die Kinder bei den Hausaufgaben häufig prokrastinieren und wenig Ausdauer zeigen, was zu familiären Konflikten führen kann (Bryan et al., 2001). Nicht selten sind aufgrund der familiären Häufung von Lernstörungen (Schulte-Körne et al., 2006) ein oder beide Eltern selbst von Lernproblemen betroffen, wodurch sie ihr Kind möglicherweise nur eingeschränkt unterstützen können. Dies wiederum kann sich negativ auf die elterliche Selbstwirksamkeit auswirken (Bonifacci, Montuschi, Lami \& Snowling, 2013). Daher ist es ratsam, bereits zu Beginn der Lernstörungsdiagnostik die Eltern-Kind-Interaktion beim Erledigen der Hausaufgaben und dem häuslichen Lernen zu explorieren.

Vor diesem Hintergrund verwundert, dass bislang nur wenig erforscht ist, wie sich Eltern von Kindern mit LS in die Hausaufgabensituation einbringen und das häusliche Üben gestalten. Diese Frage ist nicht trivial, denn es ist bekannt, dass Eltern mit vermehrtem Unterstützungsverhalten auf die schulischen Probleme ihres Kindes zu reagieren versuchen (Levin et al., 1997; Silinskas, Niemi, Lerkkanen \& Nurmi, 2012). Weitestgehend ungeklärt ist jedoch, wie sich die Eltern dabei einbringen (vgl. Silinskas et al., 2012) und wie gut das gemeinsame Lernen funktioniert. Ein Ziel dieser Studie war daher zu untersuchen, ob sich Eltern von Kindern mit LS anders in die Hausaufgabensituation einbringen als Eltern von Kindern ohne LS.

Bisherige Studien (z.B. Núňez et al., 2015) legen außerdem nahe, dass das elterliche Unterstützungsverhal- ten je nach Klassenstufe variiert, denn Kinder in der Grundschule erhalten nach eigenen Angaben häufiger Hausaufgabenhilfe von ihren Eltern als Kinder der Sekundarstufe (Gerber \& Wild, 2009). Auch der Schulzweig spielt eine Rolle, denn auf dem Gymnasium wird von mehr elterlicher Lernunterstützung und Autonomie berichtet als auf der Hauptschule (Gerber \& Wild, 2009). Allerdings beruhen die meisten dieser Schlussfolgerungen auf Querschnittsstudien. Nur für den frühen Grundschulbereich liegen vereinzelt Längsschnittstudien (z. B. Levin et al., 1997; Silinskas et al., 2012) vor, die Veränderungen der elterlichen Lernunterstützung über mehrere Schuljahre hinweg betrachteten. Die Eltern der vorliegenden Studie wurden daher wiederholt zu ihrer familiären Hausaufgabenpraxis befragt, wodurch die längsschnittliche Variabilität der elterlichen Lernunterstützung untersucht werden konnte. Fokussiert wurden dabei angesichts ihrer Zusammenhänge mit der Leistungsentwicklung und -motivation (Dumont et al., 2012) folgende Aspekte:

a. die elterliche Kontrolle der Hausaufgaben, d.h. ob die Eltern die Richtigkeit der Aufgaben überprüfen und inwiefern sie sich während der Bearbeitung der Hausaufgaben einmischen und deren Erledigung überwachen,

b. die elterliche Hilfe bei Problemen mit den Hausaufgaben, d.h. ob die Eltern bei auftretenden Schwierigkeiten gemeinsam mit dem Kind nach einer Lösung suchen, es dabei kompetent anleiten und dem Kind helfen, negative Emotionen zu regulieren,

c. die familiäre Belastung, d.h. ob das Erledigen der Hausaufgaben häufig mit Konflikten einhergeht und inwiefern die Familie beim häuslichen Lernen überfordert ist.

Weniger im Fokus stand bisher die elterliche Erwartung an die Lernbemühungen des Kindes. Mittlerweile ist jedoch bekannt, dass Eltern in westlichen Kulturen schulischen Erfolg ihrer Kinder zumeist auf deren Begabung und auf Anstrengung attribuieren und Misserfolg auf mangelnde Anstrengung (Enlund, Aunola \& Nurmi, 2015; Rytkönen, Aunola \& Nurmi, 2005). Nach der Attributionstheorie von Weiner (2014) handelt es sich bei der Attribution auf Anstrengung um eine internale, zeitlich instabile und kontrollierbare Ursache. Demnach wäre die Anstrengungsbereitschaft grundsätzlich beeinflussbar durch das Kind. Daher stellt sich die Frage nach der elterlichen Anstrengungserwartung bei Kindern mit LS, also inwiefern die Eltern Fleiß und Ausdauer bei den häuslichen Lernaktivitäten einfordern und als Garant für schulischen Erfolg sehen. Dies ist auch deshalb relevant, weil Lernstörungen nach der ICD-10 (Dilling, Mombour \& Schmidt, 2015) nicht primär die Folge einer geringen Anstrengungsbereitschaft sind. Kindern ohne LS mag es ge- 
lingen, auftretende Schwierigkeiten bei den Hausaufgaben mit Geduld und Konzentration zu meistern. Die massiven Lernprobleme bei Lernstörungen lassen sich hingegen nicht durch mangelnden Fleiß erklären, sondern gehen primär auf umschriebene Probleme in der neurokognitiven Informationsverarbeitung zurück (Lonnemann et al., 2011; Schulte-Körne et al., 2006).

\section{Elterliche Lernunterstützung bei Kindern mit LS}

Die häuslichen Lernaktivitäten in Familien von Kindern mit LS sind bisher kaum untersucht. Studien mit unausgelesenen Stichproben berichten jedoch von einem negativen Zusammenhang zwischen dem schulischen Leistungsniveau der Kinder und der elterlichen Hausaufgabenkontrolle: Eltern von leistungsschwachen Kindern überwachen die Hausaufgaben demnach intensiver und mischen sich häufiger „ungefragt“ ein. Dieser Effekt wurde sowohl zu Schulanfang (Silinskas et al., 2012) als auch in der Mittelstufe (Dumont et al., 2012; Núňez et al., 2015) nachgewiesen.

Hinsichtlich der elterlichen Hilfe bei Hausaufgabenproblemen ist die Befundlage heterogen. Im Grundschulbereich ergab sich ein negativer Zusammenhang zwischen kindlichem Leistungsniveau und elterlicher Hilfe (Silinskas et al., 2012). Zwei Studien aus der Mittelstufe (Dumont et al., 2012; Núňez et al., 2015) wiederum stellten keinen Zusammenhang zwischen dem kindlichen Leistungsniveau und dem Ausmaß elterlicher Hilfe fest.

In Bezug auf die familiäre Belastung zeigen Befunde, dass Familien mit leistungsschwachen Grundschulkindern mehr negativen Affekt und Belastung bei den Hausaufgaben erleben als Familien mit leistungsstärkeren Kindern (Levin et al., 1997; Silinskas, Kiuru, Aunola, Lerkkanen \& Nurmi, 2015). Auch empfinden Mütter von Kindern mit diagnostizierter LS mehr Frustration bei der Hausaufgabenhilfe und sind häufiger resigniert als Mütter von Kindern ohne LS (Bryan et al., 2001).

Für die elterliche Anstrengungserwartung fanden $\mathrm{Na}-$ tale und Kollegen (2008), dass Mütter von Erstklässlern mit einem familiären Risiko für Lese-Rechtschreibstörung (LRS) den schulischen Misserfolg ihrer Kinder weniger auf mangelnde Anstrengung attribuieren als die Mütter der Vergleichsgruppe. Ob Eltern dieses Attributionsmuster auch bei älteren Kindern mit einer manifesten LS anwenden, wurde noch nicht untersucht.

\section{Wie verändern Eltern ihre Lernunterstützung, wenn die Kinder älter werden?}

Auch das Alter des Kindes scheint zu beeinflussen, wie sich die Eltern beim häuslichen Üben einbringen. So stellten Levin et al. (1997) über die ersten drei Grundschuljahre einen Rückgang der elterlichen Hauaufgabenunterstützung fest, ohne jedoch zwischen verschiedenen Verhaltensdimensionen zu differenzieren. Im Gegensatz dazu untersuchten Silinskas et al. (2015) verschiedene Aspekte elterlicher Unterstützung und fanden für die Hausaufgabenkontrolle und für die Hilfe bei Problemen eine Abnahme über die Grundschulzeit. Interessanterweise nahmen zeitgleich das mütterliche Belastungserleben und der negative Affekt während der Hausaufgaben zu. $\mathrm{Zu}$ leicht anderen Ergebnissen kamen Núňez et al. (2015), die drei Alterskohorten in der Mittelstufe verglichen. Ein negativer Zusammenhang mit dem Alter der Kinder wurde hier nur für die elterliche Kontrolle der Hausaufgaben gefunden, nicht jedoch für Hilfe bei Problemen.

Für die Anstrengungserwartung wurden längsschnittliche Veränderungen von Natale und Kollegen (2008) untersucht: Im Verlauf der ersten Klasse blieb die Anstrengungserwartung der Mütter auf konstantem Niveau und wies eine sehr hohe Rangordnungsstabilität auf. Ebenso bestanden keine Unterschiede in den differenziellen Stabilitäten in Abhängigkeit eines familiären LRS-Risikos. Bei einem deutlich größeren Untersuchungszeitraum scheinen sich hingegen durchaus Veränderung in der elterlichen Anstrengungsattribution einzustellen: Enlund und Kollegen (2015) fanden bei einem Vergleich zwischen der ersten und der neunten Klasse zwar intraindividuell ebenfalls keine nennenswerten Veränderungen im mittleren Ausmaß der elterlichen Anstrengungserwartung, dafür aber zum Teil Unterschiede in der interindividuellen Stabilität dieser Attributionen. Auch die Studie von Silinskas et al. (2012) hat sich mit dem mittleren Trend über die Zeit und der Rangordnungsstabilität beschäftigt. Ihre Befunde zur elterlichen Hilfe und elterlichen Kontrolle der Hausaufgaben sprechen hingegen dafür, dass das elterliche Unterstützungsverhalten zeitlich stabil ist.

\section{Unterschiede zwischen Vätern und Müttern}

In der vorliegenden Studie wurde zusätzlich untersucht, ob sich Väter und Mütter in ihrer Hausaufgabenunter- 
stützung unterscheiden. Erste Studien mit Fokus auf den Schulbeginn (z. B. Silinskas et al., 2012) sprechen dafür, dass Mütter die Hausaufgaben etwas häufiger kontrollieren und öfter bei Problemen unterstützen als Väter. Auch ist ihr Engagement zeitlich stabiler und das Ausmaß ihres Unterstützungsverhaltens stärker durch das Leistungsniveau des Kindes beeinflusst. In Bezug auf die Kausalattributionen berichten Cote und Azar (1997), dass Mütter schulischen Erfolg stärker auf Anstrengung attribuieren als Väter, während dieser Geschlechtseffekt bei der Kausalattribution für schulischen Misserfolg ausblieb.

\section{Hypothesen}

Es wird davon ausgegangen, dass die Eltern von Kindern mit LS (1) die Hausaufgaben und das häusliche Lernen stärker überwachen, (2) ein erhöhtes Belastungserleben bei der Hausaufgabenunterstützung aufweisen und (3) eine geringere Anstrengungserwartung zeigen als die Eltern der Vergleichsgruppe. In Bezug auf die längsschnittlichen Verläufe wird sowohl für die Eltern von Kindern mit als auch ohne LS erwartet, dass (4) die Häufigkeit der Hausaufgabenkontrolle über den getesteten 2-Jahresverlauf zurückgeht, während sich (5) das mittlere Ausmaß der elterlichen Anstrengungserwartung nicht verändert.

\section{Methodik}

\section{Durchführung}

Die Kinder wurden über Schulen und (Erziehungs-)Beratungsstellen im Großraum Frankfurt am Main rekrutiert. Bei einer Rekrutierung über Schulen wurde zunächst eine standardisierte Lernstanderfassung durch das Projektteam durchgeführt, um zu prüfen, ob das Kind die für die Studie relevanten Einschlusskriterien erfüllte. Bei Kindern mit diagnostizierter LS, die über Beratungsstellen rekrutiert wurden, fand eingangs nur dann eine Überprüfung des Lernstandes statt, wenn das vorliegende diagnostische Gutachten unzureichende Informationen über den mit standardisierten Leistungstests erfassten Lernstand enthielt. Die Familien wurden anschließend jährlich im Frühjahr und im Herbst zu den Erhebungen eingeladen und der begleitende Elternteil füllte den Fragebogen zur familiären Lern- und Hausaufgabenpraxis aus. Der Lernstand der Kinder wurde zwei Jahre nach Studienbeginn erneut überprüft.

\section{Stichprobe}

Von den 82 Kindern erfüllten 47 Kinder die für die Studie gewählten Einschlusskriterien für LS: (a) Sie erbrachten unterdurchschnittliche Leistungen im Lesen, Rechtschreiben und/oder Rechnen $(\mathrm{T}<40$ ), (b) ihre nonverbale Intelligenz war mit IQ $\geq 85$ mindestens durchschnittlich und (c) es lag eine IQ-Diskrepanz in Höhe von wenigstens 10 T-Wertpunkten vor. Diesen Kriterien entsprechend zeigten 35 Kinder eine Lese-Rechtschreibstörung (F81.0), acht Kinder eine Rechenstörung (F81.2) und vier Kinder eine kombinierte Störung schulischer Fertigkeiten (F81.3). Kinder der Vergleichsgruppe mussten eine unbeeinträchtigte Intelligenz (IQ > 85) aufweisen und in allen drei Lernbereichen mindestens durchschnittliche Werte $(T \geq 42)$ erzielen. Die Kinder mit LS erzielten bei beiden Lernstanderfassungen deutlich schwächere Werte als die Kinder der Vergleichsgruppe (Tab. 1). Bedeutsame Gruppenunterschiede bestanden außerdem in der Deutsch- und Mathematiknote der vierten Klasse, wobei die Rechtschreibleistung bei 16 Kindern (34\%) und das Kopfrechnen bei zwei Kindern mit LS (4\%) aufgrund von Notenschutz bei der Benotung unberücksichtigt blieben.

Die Gruppen unterschieden sich nicht im Geschlecht, $\chi^{2}(1)=2.07, p=.15$. Zum ersten Messzeitpunkt (MZP) befanden sich 71 Kinder im zweiten Halbjahr der 4. Klasse; vier Kinder waren in der 3. Klasse und sieben bereits in der 5. Klasse. Nach dem Übergang auf die weiterführende Schule besuchten die Kinder mit LS mehrheitlich eine Gesamtschule und die Kinder der Vergleichsgruppe überwiegend ein Gymnasium (Tab. 1). Von den Kindern mit LS liegen zusätzlich Informationen zur Teilnahme an Fördermaßnahmen vor: 29 Kinder (62\%) nahmen am schulischen Förderunterricht teil; eine außerschulische Lerntherapie besuchten 27 Kinder (57\%); für fünf Kinder fehlen die Angaben. Außerdem gaben 31 Eltern von Kindern mit LS (66\%) an, dass wenigstens ein Elternteil als Kind ebenfalls Lernschwierigkeiten hatte.

Der Fragebogen zur familiären Lern- und Hausaufgabensituation wurde mehrheitlich von der Mutter $(n=65$; $79 \%$ ) ausgefüllt. Die Rücklaufquote betrug $84 \%$ zum zweiten, $73 \%$ zum dritten und $63 \%$ zum vierten MZP und unterschied sich nicht bedeutsam zwischen den beiden Gruppen, MZP2: $\chi^{2}(1)<1, p=.74$, MZP3: $\chi^{2}(1)=1.45, p=$ .23, MZP4: $\chi^{2}(1)=1.69, p=.19$. Auch bestanden in den Diagnosevariablen keine Unterschiede in Abhängigkeit der Rücklaufquote, d.h. die Lernleistungen waren vergleichbar zwischen den Kindern mit und ohne fehlende Daten. 
Tabelle 1. Deskriptive Angaben zur Stichprobe

\begin{tabular}{|c|c|c|c|c|c|c|}
\hline & \multicolumn{2}{|c|}{ Kinder der Vergleichsgruppe } & \multicolumn{2}{|c|}{ Kinder mit Lernstörung } & \multirow[b]{2}{*}{$F$} & \multirow[b]{2}{*}{$p$} \\
\hline & M & $S D$ & M & $S D$ & & \\
\hline Alter & 121.27 & 5.94 & 124.02 & 5.72 & 3.28 & .080 \\
\hline \multicolumn{7}{|l|}{ Lernstanderfassung I } \\
\hline Intelligenz ${ }^{a}$ & 109.97 & 12.50 & 105.68 & 10.59 & 2.82 & .100 \\
\hline Lesen $^{b}$ & 59.94 & 9.66 & 40.51 & 10.02 & 73.43 & $<.001$ \\
\hline Rechtschreiben ${ }^{\mathrm{b}}$ & 57.91 & 10.60 & 36.57 & 7.36 & 102.67 & $<.001$ \\
\hline Rechnen $^{b}$ & 57.89 & 10.51 & 44.47 & 10.72 & 28.32 & $<.001$ \\
\hline \multicolumn{7}{|l|}{ Lernstanderfassung II } \\
\hline Lesen $^{b}$ & 56.90 & 9.64 & 38.83 & 8.32 & 64.77 & $<.001$ \\
\hline Rechtschreiben ${ }^{b}$ & 54.66 & 10.36 & 35.83 & 8.82 & 61.73 & $<.001$ \\
\hline Rechnen $^{b}$ & 51.57 & 11.43 & 43.22 & 9.12 & 11.07 & .001 \\
\hline Deutschnote (4. Klasse) & 2.10 & 0.76 & 3.11 & 0.75 & 32.65 & $<.001$ \\
\hline \multirow[t]{2}{*}{ Mathematiknote (4. Klasse) } & 2.23 & 0.88 & 2.93 & 0.95 & 10.64 & .002 \\
\hline & $n$ & $\%$ & $n$ & $\%$ & & \\
\hline \multicolumn{7}{|l|}{ Geschlecht } \\
\hline Jungen & 19 & 54 & 29 & 62 & & \\
\hline Mädchen & 16 & 46 & 18 & 38 & & \\
\hline \multicolumn{7}{|l|}{ Schultyp ${ }^{c}$} \\
\hline Hauptschule & 0 & 0 & 2 & 4 & & \\
\hline Realschule & 3 & 9 & 9 & 19 & & \\
\hline Gymnasium & 24 & 69 & 7 & 15 & & \\
\hline Gesamtschule & 4 & 11 & 23 & 49 & & \\
\hline fehlende Angabe & 4 & 11 & 6 & 13 & & \\
\hline
\end{tabular}

Anmerkungen: Tests zur Lernstanderfassung I: Intelligenz = Columbia Mental Maturity Scale (CMM 1-3), Rechtschreiben = Deutscher Rechtschreibtest für erste und zweite Klassen (DERET 1-2+), Lesen = Würzburger Leise Leseprobe (WLLP), Rechnen = Deutscher Mathematiktest für zweite Klassen (DEMAT 2 +); bei 14 Kindern lag ein externes diagnostisches Gutachten mit standardisierter Leistungsdiagnostik vor. Tests zur Lernstanderfassung II (ca. zwei Jahre nach Lernstand I): Lesen = Ein Leseverständnistest für Erst- bis Sechstklässler (ELFE 1-6), Rechtschreiben = Deutscher Rechtschreibtest für vierte Klassen (DERET 4) bzw. Diagnostischer Rechtschreibtest für 5. Klassen (DRT 5), Rechnen = Deutscher Mathematiktest für vierte Klassen (DEMAT 4) bzw. Rechenfertigkeiten- und Zahlenverarbeitungs-Diagnostikum für die 2. bis 6 . Klasse (RZD 2-6); ${ }^{a} \mid$ Q-Werte $(M=100, S D=15) ;{ }^{b} T$-Werte $(M=50, S D=10) ;{ }^{\circ} A n g a b e n$ zum Schulzweig fehlen bei vier Kindern der Vergleichsgruppe und bei sechs Kindern mit LS

\section{Fragebogen zur familiären Hausaufgabenpraxis}

Die Skala Kompetente Hilfe erfragte in fünf Items, inwiefern sich die Eltern bei Lern- und Hausaufgabenproblemen aktiv an deren Lösung beteiligen sowie die wahrgenommene Kompetenz der Eltern ihrem Kind bei Problemen helfen zu können (z. B. Bei Schulschwierigkeiten überlegen mein Kind und ich gemeinsam, wie wir die Probleme lösen können.). Die interne Konsistenz der Skala war mit McDonald's $\omega=.75$ für die Grundlagenforschung noch akzeptabel.

Die fünf Items der Skala Kontrolle erfragten das Ausmaß, in dem die Eltern das Erledigen der Hausaufgaben ihrer Kinder aktiv steuern sowie deren Richtigkeit kontrollieren (z. B. Ich achte sehr darauf, dass mein Kind immer alle Hausaufgaben richtig macht). Die Skala hatte eine akzeptable interne Konsistenz (McDonald's $\omega=.77$ ).
Die Skala Belastung erfasste in sechs Items, inwiefern es beim Erledigen der Hausaufgaben bzw. aufgrund der schulischen Leistungen zu Streit in der Familie kommt und wie sehr die Familie durch die Probleme belastet ist (z. B. Die schulischen Leistungen meines Kindes belasten den Familienalltag). Die Skala hatte eine gute interne Konsistenz (McDonald's $\omega=.82$ ).

Die vier Items der Skala Anstrengungserwartung erfassten das Ausmaß, in dem Eltern schulischen Erfolg auf Anstrengung und Übung attribuieren (z. B. Jedes Kind kann gut in der Schule sein, wenn es genug übt) bzw. fehlende Anstrengung für schulische Misserfolge verantwortlich machen. Die Skala zeigte eine akzeptable interne Konsistenz (McDonald's $\omega=.75$ ).

Die Fragen sind teils angelehnt an Items anderer Studien (Bruder, 2006; Otto, 2007) und wurden anhand einer vierstufigen Likert-Skala (1 Punkt: trifft nicht $z u$ bis 4 Punkte: trifft vollkommen $z u$ ) eingeschätzt. Eine erste 
Version des Fragebogens wurde in einer Online-Studie von Roth (2010) entwickelt und an 106 Eltern pilotiert.

\section{Statistische Auswertung}

Für jede Skala wurden latente Veränderungsmodelle mit dem robusten Maximum Likelihood Schätzer (MLR) in Mplus 7.11 spezifiziert. Die Skalenrohwerte flossen als manifeste Variablen in die Berechnung ein. Modelliert wurde ein latenter Interceptfaktor, der das Ausgangsniveau im Unterstützungsverhalten zum ersten MZP widerspiegelt, sowie ein linearer Wachstumsfaktor, der die lineare Veränderung über den zweijährigen Studienverlauf angibt (vgl. Abb. 1). Erwies sich die Varianz des Interceptbzw. des Wachstumsfaktors als signifikant, wurden im nächsten Schritt die Faktoren Lernstörung und Elternteil zur Erklärung der interindividuellen Unterschiede aufgenommen. Ein Model Fit wurde als gut bewertet bei einem Comparative Fit Index (CFI) > .95, einem Root Mean Square Error of Approximation (RMSEA) $<.06$ und einem nicht signifikanten $\chi^{2}$-Wert.

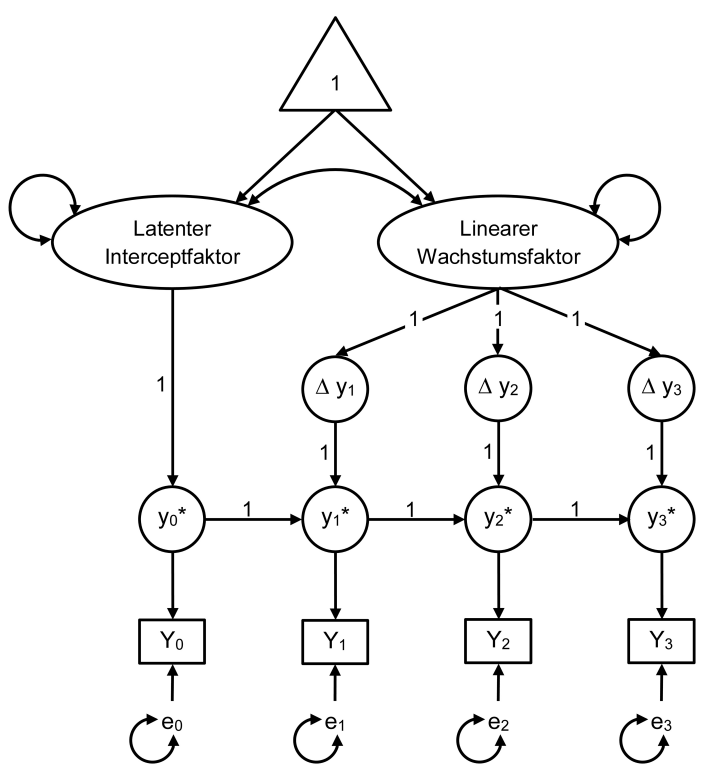

Abbildung 1. Latentes Veränderungsmodell mit linearem Wachstumsfaktor.

\section{Ergebnisse}

\section{Korrelationen}

Erwartungskonform ergaben sich hoch-positive IntraSkalen-Korrelationen über die vier MZP (Tab. 2), was für eine gewisse zeitliche Stabilität des elterlichen Unterstüt- zungsverhaltens spricht. Die Inter-Skalen-Korrelationen fielen hingegen schwach aus. Lediglich zwischen den Skalen Belastung und Kompetente Hilfe war ein Großteil der Korrelationen über die Zeit hinweg signifikant negativ: Eltern, die sich wenig kompetent fühlten, ihr Kind bei den Hausaufgaben zu unterstützen, nahmen die Hausaufgabensituation als belastender wahr als Eltern, die ihr eigenes Unterstützungsverhalten als kompetent erlebten. Ebenso war ein Großteil der Korrelationen zwischen den Skalen Belastung und Kontrolle sowie zwischen den Skalen Anstrengungserwartung und Kompetente Hilfe schwach positiv.

\section{Latente Veränderungsmodelle}

Für die Skala Kompetente Hilfe war der Modellfit gut, $\chi^{2}(8)=12.91, p=.12$, CFI $=.94$, RMSEA $=.09$. Beim Interceptfaktor war sowohl der Mittelwert, $\mu=14.56, S E=$ $0.34, p<.001$, als auch die Varianz, $\sigma^{2}=6.17, S E=1.60$, $p<.001$, signifikant. Aus der Höhe des Mittelwertes lässt sich ableiten, dass die Eltern ihr Kompetenzerleben zum ersten MZP als eher hoch einschätzten. Die signifikante Varianz weist auf interindividuelle Unterschiede zwischen den Eltern hin. Im nächsten Schritt wurden daher die beiden Prädiktoren in das Modell aufgenommen. Der Modellfit erwies sich dabei als sehr gut, $\chi^{2}(15)=15.55, p=$ .41 , CFI $=.99$, RMSEA $=.02$, und erbrachte ein signifikantes Ergebnis für den Faktor Lernstörung, $B=-1.54$, $S E=0.63, \beta=-.30, p=.02$. Eltern von Kindern mit LS fühlten sich demnach beim ersten MZP weniger kompetent, ihr Kind bei den Hausaufgaben unterstützen zu können als Eltern der Vergleichsgruppe. Demgegenüber machte es für das Kompetenzerleben keinen Unterschied, ob der Vater oder die Mutter den Fragebogen ausgefüllt hat, $B=0.09, S E=0.79, \beta=.01, p=.91$. Für den Wachstumsfaktor erwies sich weder der Mittelwert, $\mu=-0.07, S E$ $=0.13, p=.61$, noch die Varianz, $\sigma^{2}=0.11, S E=0.21, p=$ .69 , als signifikant. So kam es im Verlauf der Studie weder zu nennenswerten mittleren Veränderungen in der elterlichen Hilfe noch lagen interindividuell unterschiedliche Verläufe vor.

Auch für die Skala Kontrolle war der Modellfit gut, $\chi^{2}(8)=12.98, p=.11$, CFI $=.96$, RMSEA $=.09$. Beim Interceptfaktor waren erneut der Mittelwert, $\mu=10.82$, $S E=0.37, p<.001$, und die Varianz, $\sigma^{2}=8.24, S E=1.36$, $p<.001$, signifikant. Die Höhe des Mittelwertes weist auf ein mittleres Ausmaß elterlicher Hausaufgabenkontrolle zum ersten MZP hin. Die signifikante Varianz gibt an, dass sich die Eltern im Kontrollverhalten voneinander unterschieden. Durch die Prädiktoren konnte ein bedeutsamer Anteil dieser Varianz erklärt werden: Eltern von Kindern mit LS kontrollierten die Hausaufgaben ihrer 
Tabelle 2. Deskriptive Statistiken zu den Skalen des Elternfragebogens

\begin{tabular}{|c|c|c|c|c|c|c|c|c|c|c|c|c|c|c|c|c|}
\hline Skalen & 1. & 2. & 3. & 4. & 5. & 6. & 7. & 8. & 9. & 10. & 11. & 12. & 13. & 14. & 15. & 16. \\
\hline 1. Anstrengungserwartung t1 & - & & & & & & & & & & & & & & & \\
\hline 2. Anstrengungserwartung t2 & .70 & - & & & & & & & & & & & & & & \\
\hline 3. Anstrengungserwartung t3 & .77 & .76 & - & & & & & & & & & & & & & \\
\hline 4. Anstrengungserwartung t4 & .70 & .76 & .71 & - & & & & & & & & & & & & \\
\hline 5. Belastung t1 & .01 & .01 & -.15 & -.01 & - & & & & & & & & & & & \\
\hline 6. Belastung t2 & -.11 & -.06 & -.18 & -.07 & .62 & - & & & & & & & & & & \\
\hline 7. Belastung t3 & .01 & -.01 & -.10 & -.06 & .73 & .71 & - & & & & & & & & & \\
\hline 8. Belastung t4 & .01 & -.06 & -.08 & -.05 & .59 & .72 & .87 & - & & & & & & & & \\
\hline 9. Hilfe t1 & .22 & .32 & .24 & .37 & -.11 & -.13 & -.26 & -.23 & - & & & & & & & \\
\hline 10. Hilfe t2 & .07 & .24 & .18 & .16 & -.22 & -.29 & -.40 & -.36 & .63 & - & & & & & & \\
\hline 11. Hilfe t3 & .10 & .19 & .25 & .27 & -.17 & -.17 & -.30 & -.37 & .51 & .74 & - & & & & & \\
\hline 12. Hilfe t4 & .15 & .23 & .23 & .22 & -.03 & -.26 & -.33 & -.45 & .60 & .75 & .78 & - & & & & \\
\hline 13. Kontrolle t1 & -.06 & -.07 & -.15 & .02 & .40 & .20 & .27 & .23 & .17 & -.06 & -.04 & .07 & - & & & \\
\hline 14. Kontrolle t2 & .17 & .29 & .21 & .15 & .23 & .26 & .32 & .21 & .19 & .19 & .24 & .29 & .62 & - & & \\
\hline 15. Kontrolle t3 & .11 & .05 & .06 & .03 & .26 & .15 & .27 & .20 & .11 & .12 & .24 & .28 & .73 & .77 & - & \\
\hline 16. Kontrolle t4 & -.02 & .03 & .07 & .08 & .29 & -.04 & .15 & .05 & .12 & .06 & .26 & .32 & .68 & .66 & .80 & - \\
\hline $\bar{M}$ & 9.90 & 10.08 & 10.26 & 10.44 & 9.94 & 9.51 & 9.09 & 8.66 & 14.56 & 14.50 & 14.43 & 14.37 & 10.82 & 10.50 & 10.19 & 9.87 \\
\hline$S D$ & 2.34 & 2.29 & 2.26 & 2.23 & 3.28 & 2.86 & 2.67 & 2.72 & 2.48 & 2.55 & 2.67 & 2.81 & 2.87 & 2.84 & 2.83 & 2.83 \\
\hline
\end{tabular}

Anmerkungen: $r>.21$ bei einem zweiseitigem $\alpha$-Niveau von .05 signifikant

Kinder stärker als die Eltern der Vergleichsgruppe, $B=$ 1.30, $S E=0.64, \beta=.23, p=.04$. Aber es machte erneut keinen Unterschied, welches Elternteil den Fragebogen ausgefüllt hat, $B=0.79, S E=0.76, \beta=.11, p=.30$. Der Modellfit dieses Prädiktorenmodells war sehr gut, $\chi^{2}(15)=$ $14.55, p=.48, \mathrm{CFI}=1.00$, RMSEA $=.00$. Für den Wachstumsfaktor erwies sich lediglich der Mittelwert, $\mu=-0.31$, $S E=0.11, p=.005$, jedoch nicht die Varianz, $\sigma^{2}=0.05, S E$ $=0.16, p=.78$, als signifikant. Der negative Mittelwert des Wachstumsfaktors gibt an, dass es über den Untersuchungszeitraum von zwei Jahren einen mittleren Rückgang im elterlichen Kontrollverhalten gab.

Für die Skala Belastung war der Modellfit zunächst nur mäßig, $\chi^{2}(8)=25.81, p=.001, \mathrm{CFI}=.84$, RMSEA $=.17$, weil die Residualvarianz zum zweiten MZP nicht mit den anderen invariant gesetzt werden konnte. Eine mögliche Erklärung dafür ist, dass ein Großteil der Kinder auf die weiterführende Schule eingeschult wurde und dieser MZP daher von den Eltern anders bewertet wurde als die anderen MZP. Nach Lockerung dieser Restriktion war der Fit sehr gut, $\chi^{2}(7)=7.04, p=.43, \mathrm{CFI}=1.00$, RMSEA $=$ .009. Beim Interceptfaktor war der Mittelwert zwar signifikant, aber von geringem Ausmaß, $\mu=9.94, S E=$ $0.39, p<.001$, was im Mittel auf eine eher niedrige Belastung der Eltern zum ersten MZP hinweist. Die signifikante Varianz des Interceptfaktors, $\sigma^{2}=10.77, S E=2.64$, $p<.001$, zeigt interindividuelle Unterschiede auf: Während sich manche Eltern bei Studienbeginn durch die
Hausaufgabensituation ihrer Kinder kaum belastet fühlten, berichteten andere ein höheres Konfliktpotenzial. Ein Teil dieser Varianz konnte durch den Faktor Lernstörung, $B=1.76, S E=.79, \beta=.27, p=.03$, jedoch nicht durch den Faktor Elternteil, $B=-1.00, S E=0.89, \beta=-.12, p=.26$; aufgeklärt werden: Eltern von Kindern mit LS waren zu Studienbeginn belasteter als Eltern der Vergleichsgruppe. Der Fit dieses Prädiktorenmodells war sehr gut, $\chi^{2}(11)=$ $16.43, p=.13$, CFI $=.96$, RMSEA $=.08$. Beim Wachstumsfaktor waren sowohl der Mittelwert, $\mu=-0.43, S E=$ $0.13, p=.001$, als auch die Varianz, $\sigma^{2}=0.69, S E=0.26$, $p=.007$, signifikant. Der negative Mittelwert des Wachstumsfaktors bedeutet, dass die Belastung der Eltern über den zweijährigen Studienverlauf im Mittel zurückging. Die signifikante Varianz des Wachstumsfaktors weist jedoch darauf hin, dass dies nicht für alle Eltern gleichermaßen galt. Das Ausmaß interindividueller Unterschiede im Rückgang der Belastung hing jedoch weder mit dem Vorliegen einer LS, $B=-0.06, S E=0.26, \beta=-.04, p=.81$, noch dem Geschlecht des Elternteils, $B=-0.09$, $S E=$ $0.40, \beta=-.05, p=.81$, zusammen.

Für die Skala Anstrengungserwartung war der Modellfit gut, $\chi^{2}(8)=6.60, p=.58, \mathrm{CFI}=1.00$, RMSEA $=.00$. Beim Interceptfaktor wurde sowohl der Mittelwert, $\mu=9.90$, $S E=0.30, p<.001$, als auch die Varianz, $\sigma^{2}=5.46, S E=$ 1.00, $p<.001$, signifikant. Der eher niedrige Mittelwert zeigt, dass die Eltern im Mittel ein niedriges latentes Niveau aufwiesen, schulischen Erfolg auf Anstrengung zu 
attribuieren. Die signifikante Varianz des Interceptfaktors gibt an, dass manche Eltern schulischen Erfolg beim ersten MZP stärker auf Anstrengung attribuierten als andere Eltern. Von den beiden Prädiktoren wurden sowohl der Faktor Lernstörung signifikant, $B=-1.98, S E=0.50, \beta=$ -.43, $p<.001$, als auch der Faktor Elternteil, $B=1.26, S E=$ $0.64, \beta=.23, p=.049$. Eltern von Kindern mit LS attribuierten schulischen Erfolg demnach seltener auf Anstrengung und Übung. Außerdem berichteten Mütter ein höheres Ausmaß an Anstrengungserwartung als Väter. Der Fit dieses Prädiktorenmodells war sehr gut, $\chi^{2}(15)=$ $9.39, p=.86$, CFI $=1.00$, RMSEA $=.00$. Beim Wachstumsfaktor wurde weder der Mittelwert, $\mu=0.18, S E=$ $0.10, p=.07$, noch die Varianz, $\sigma^{2}=0.02, S E=0.10, p=$ .81 , signifikant.

\section{Diskussion}

\section{Wie unterscheidet sich die Hausaufgaben- situation bei Kindern mit und ohne LS?}

Diesbezüglich zeigte sich, dass die wahrgenommene Kompetenz der Eltern, ihrem Kind bei Hausaufgabenproblemen weiterhelfen zu können, deutlich geringer ausgeprägt war, wenn das Kind eine LS aufwies. Dies rührt möglicherweise daher, dass immerhin $66 \%$ dieser Eltern berichteten ebenfalls Lernschwierigkeiten in der Schule gehabt zu haben. Da Personen mit LS in der Kindheit meist auch im Erwachsenenalter noch schwächere Leistungen in dem betroffenen Lernbereich erzielen (z. B. Schulte-Körne, Deimel, Jungermann \& Remschmidt, 2003), ist naheliegend, dass dies bei den Eltern mit Sorgen darüber einhergeht, ihre Kinder beim Lernen nicht gut unterstützen zu können. Alternativ wäre aber auch denkbar, dass die Eltern von Kindern mit LS durchaus kompetente Hausaufgabenhilfe zeigen, diese aber aufgrund der massiven Lernrückstände der Kinder nicht fruchtet, was $\mathrm{zu}$ einem reduzierten Kompetenzerleben der Eltern führt. Um diesen beiden Erklärungsmöglichkeiten weiter nachzugehen, wären künftig direkte Beobachtungen der Eltern-Kind-Interaktion sinnvoll, um zwischen wahrgenommener und tatsächlicher Kompetenz zu unterscheiden.

Auch überwachten die Eltern von Kindern mit LS die Richtigkeit der Hausaufgaben mehr und mischten sich stärker (ungefragt) in deren Erledigung ein als die Eltern der Vergleichsgruppe. Dies ist im Einklang mit Studien an unausgelesenen Stichproben (Núňez et al., 2015), die einen negativen Zusammenhang zwischen dem kindlichen Leistungsniveau und der elterlichen Hausaufgabenkontrolle berichten. Bedenkenswert an diesem Ergebnis ist jedoch, dass sich in einer Längsschnittstudie von Ng et al. (2004) ein erhöhtes Ausmaß elterlicher Kontrolle negativ auf die Motivation der Kinder auswirkte und dieser Effekt bei leistungsschwachen Kindern deutlich stärker ausgeprägt war als bei leistungsstarken. Übermäßige Kontrolle führe nach den Autoren insbesondere bei Leistungsschwachen dazu, dass sich die Kinder noch inkompetenter fühlen und in ihrem schulischen Selbstwert weiter geschwächt werden.

Ebenfalls erwartet und im Einklang mit Bryan und Kollegen (2001) sowie Bonifacci et al. (2013) wurde die familiäre Hausaufgabensituation bei Kindern mit LS als deutlich belasteter wahrgenommen als bei der Vergleichsgruppe. Dies ist relevant, weil vermehrter Streit bei den Hausaufgaben zu weniger sensitiver Hilfe und dafür zu mehr Kontrolle durch die Eltern führt (Pomerantz, Moorman \& Litwack, 2007), wodurch ein Teufelskreis entstehen kann.

Für die elterliche Anstrengungserwartung wurde erstmalig gezeigt, dass die Eltern von Kindern mit LS schulische Leistungen weniger auf Anstrengung und Übung attribuierten als die Eltern der Vergleichsgruppe. Die Eltern von Kindern mit manifester LS in unserer Studie zeigten damit ein ähnliches Attributionsmuster wie die Mütter von Erstklässlern mit einem familiären LS-Risiko aus einer Studie von Natale und Kollegen (2008). Auch wenn nun zunächst untersucht werden müsste, inwiefern sich dies auf die Hausaufgabenbearbeitung der Kinder auswirkt, lässt sich vermuten, dass das elterliche Verhalten durchaus protektiv motiviert ist, weil LS nicht einfach Folge mangelnden Fleißes sind. Das vorliegende Ergebnis scheint dafür zu sprechen, dass sich die Eltern dessen bewusst sind. Dennoch sollte dieser Befund in künftigen Studien weiter eruieret werden, da es nach der Attributionstheorie von Weiner (2014) durchaus motivationsförderlich ist, schulische Misserfolge auf internale, zeitlich instabile und kontrollierbare Ursachen wie die Anstrengung zu attribuieren.

\section{Wie verändert sich die elterliche Unterstützung über die Zeit?}

Das zweite Ziel der Studie bestand in der Analyse der zeitlichen Veränderungen. Hierzu zeigte sich hypothesenkonform, dass die Eltern das Erledigen und die Richtigkeit der Hausaufgaben mit der Zeit immer weniger überwachten. Ein solcher Effekt wurde für die weiterführende Schule bereits von Núňez et al. (2015) im Rahmen einer Querschnittstudie berichtet und konnte nun auch längsschnittlich bestätigt werden. Ein Rückgang der elterlichen Hausaufgabenkontrolle geht dabei typischerweise einher mit einer zunehmenden Selbstständigkeit 
der Kinder (Silinskas et al., 2015) und ihrem entsprechend größerem Bedürfnis nach Autonomie - insbesondere in der Pubertät.

Ebenso wurde in dieser Studie ein Rückgang in der familiären Belastung und den Eltern-Kind-Konflikten verzeichnet. Ein Befund, der sich - vor dem Hintergrund der signifikanten Zusammenhänge mit der Skala Kontrolle möglicherweise zu einem Stück weit aus der abnehmenden Involviertheit der Eltern bei den Hausaufgaben erklärt. Im Gegensatz dazu kam es zu keinen mittleren Veränderungen in den Skalen Kompetente Hilfe und Anstrengungserwartung. Während die anderen beiden Skalen ausschließlich Verhaltens- und Erlebensaspekte abfragen, stehen bei der Anstrengungserwartung und (in geringerem Maße) bei der Skala Kompetente Hilfe generelle Einstellungen und Meinungen im Vordergrund, für die anzunehmen ist, dass sie seltener kurzfristigen Veränderungen unterliegen und daher über den relativ kleinen Untersuchungszeitraum von zwei Jahren konstant bleiben.

Werden zudem interindividuelle Unterschiede in den intraindividuellen Veränderungen betrachtet, spricht unsere Studie wie die von Silinskas et al. (2012) für eine recht hohe Rangordnungsstabilität. Lediglich bei der Skala Belastung kam es zu bedeutsamen interindividuellen Unterschieden in den längsschnittlichen Veränderungen: So gab es Familien, für die eine Abnahme des Belastungserlebens über den zweijährigen Studienverlauf nicht oder nur in geringerem Maße eintrat. Interessanterweise war dieser Effekt nicht durch das Vorliegen einer LS erklärbar, obwohl das familiäre Belastungserleben zu Studienbeginn durchaus zwischen Kindern mit und ohne LS differenzierte. Zur Erklärung dieses Befundes sollte bedacht werden, dass die Gruppe mit LS in sich bereits recht heterogen war und zwar nicht nur in Bezug auf die Art der LS, sondern auch hinsichtlich extra-curricularer Förderung: Da knapp die Hälfte der Familien eine Lerntherapie in Anspruch nahm und die professionelle Anleitung von Eltern in Bezug auf das häusliche Üben oft Bestandteil von Lerntherapien ist (Multhauf \& Buschmann, 2014), wäre denkbar, dass diese Teilstichprobe an Eltern von Kindern mit LS durchaus eine Belastungsreduktion bei den Hausaufgaben erlebte, die anderen hingegen nicht, wodurch sich bei den statistischen Analysen kein eindeutiger Effekt für den Faktor Lernstörung einstellte. Aber auch weitere Erklärungsmöglichkeiten sollten in Betracht gezogen werden. Ebenso wäre möglich, dass sich Eltern unabhängig vom Vorliegen einer LS darin unterscheiden, inwiefern der Übergang auf die weiterführende Schule für sie mit Sorgen darüber einhergeht, ob sich ihr Kind auf der neuen Schule behaupten wird. Auch die Schulnoten könnten mit dem Belastungserleben zusammenhängen, denn sie vermitteln indirekt ein Feedback über den Erfolg des häuslichen Lernens. Bleiben Noten gleich oder werden gar schlechter, könnte sich dies negativ auf das Kompetenzerleben der Eltern und ihre Belastung auswirken. Denkbar wäre ebenso, dass sich Erziehungsherausforderungen in Folge der einsetzenden Pubertät in unterschiedlichem Ausmaß auf das häusliche Üben auswirken und die Familien daher unterschiedlich stark belasteten.

\section{Unterschiede zwischen Vätern und Müttern}

Hinsichtlich der Annahme, dass sich Väter und Mütter in ihrer Lernunterstützung unterscheiden, gab es weder für das Ausgangsniveau bei Studienbeginn noch über die Zeit deutliche Belege. Die insgesamt eher geringen Unterschiede zwischen Vätern und Müttern sind jedoch vor dem Hintergrund zu betrachten, dass die Elternfragebögen mehrheitlich von den Müttern ausgefüllt wurden und die Stichprobe eher klein war. So wäre denkbar, dass sich in einer größeren Stichprobe mit einem ausgeglichenen Anteil von Müttern und Vätern mehr Unterschiede zwischen den Geschlechtern zeigen. Anders als es Silinskas und Kollegen (2012) für den Schulbeginn nachweisen konnten, zeigte sich bei den teilnehmenden Müttern dieser Studie also kein stärkeres Ausmaß an Kontrolle, was möglicherweise auch an den unterschiedlichen Alterskohorten liegt. Für diese Annahme spricht der Befund von Gerber und Wild (2009), dass Väter mit steigender Klassenstufe stärker von ihren Kindern in das häusliche Üben involviert und bei Hausaufgabenproblemen um Rat gefragt werden. Dies würde erklären, wieso sich Geschlechtsunterschiede in der Hausaufgabenkontrolle zwar bei Schulanfängern finden lassen, aber nicht mehr beim Übergang auf die weiterführende Schule. Einzig für die Anstrengungserwartung gab es Unterschiede zwischen den Elternteilen, da die Mütter schulische Leistungen stärker auf Anstrengung attribuierten und daher ausdrücklicher von ihren Kindern einforderten als die Väter.

\section{Limitationen}

Einschränkend ist zu erwähnen, dass die kleine Stichprobe die Zahl an Prädiktoren im Modell einschränkte. Mit den Faktoren Lernstörung und Elternteil konnten somit nur zwei mögliche Bedingungsfaktoren für das Ausmaß elterlichen Unterstützungsverhaltens untersucht werden. Weitere mögliche Einflussfaktoren wie etwa die Zeugnisnote oder die familiären Ressourcen und der sozioökonomische Status wurden nicht berücksichtigt. Dies wäre wünschenswert gewesen, haben doch Fritzler und Wild (2019) beispielsweise jüngst festgestellt, dass kulturelle Ressourcen eine bedeutsame Rolle für das Ausmaß an 
elterlicher Kontrolle und elterlicher Hilfe bei den häuslichen Lernaktivitäten bei Kindern mit Lese-Rechtschreibstörung spielen. Ebenso wäre die Berücksichtigung des Schulübergangs in den statistischen Analysen aufschlussreich, da das elterliche Unterstützungsverhalten je nach Schulzweig unterschiedlich auszufallen scheint (Gerber \& Wild, 2009). In der vorliegenden Studie war dies nicht möglich, da die Kinder zu unterschiedlichen Zeitpunkten in die 5. Klasse wechselten. Auch erlaubt die Auskunft der Eltern keine Rückschlüsse darauf, wie die Kinder das elterliche Unterstützungsverhalten erleben. Ein Multi-Informanten-Ansatz könnte hier künftig von Mehrwert sein.

\section{Welche praktischen Implikationen lassen sich ableiten?}

Auch wenn die berichtete elterliche Kompetenz eher hoch und die Belastung eher niedrig waren, wurden deutliche Niveauunterschiede zuungunsten der Familien von Kindern mit LS deutlich. Dies unterstreicht die Notwendigkeit, die Hausaufgabensituation im diagnostischen Prozess zu thematisieren und für den Beratungsprozess zu eruieren, wie die Familien entlastet werden können. Entsprechende Elterntrainings scheinen vielversprechend. So fanden Bruder, Perels und Schmitz (2004), dass Eltern Hausaufgabenstrategien effektiv vermittelt werden können und dies sowohl zu erhöhter Selbstwirksamkeit der Eltern als auch $\mathrm{zu}$ einer Verringerung der familiären Hausaufgabenkonflikte beiträgt.

\section{Literatur}

Betz, D. \& Breuninger, H. (1998). Teufelskreis Lernstörungen (5. Aufl.). Weinheim: Psychologie Verlags Union.

Bonifacci, P., Montuschi, M., Lami, L. \& Snowling, M. J. (2013). Parents of children with dyslexia: cognitive, emotional and behavioural profile. Dyslexia, 20, 175-190. https://doi.org/10. 1002/dys.1469

Bruder, S. (2006). Förderung von Selbstregulation bei Kindern unter Einbeziehung ihrer Eltern. Berlin: Logos Verlag.

Bruder, S., Perels, F. \& Schmitz, B. (2004). Selbstregulation und elterliche Hausaufgabenunterstützung. Die Evaluation eines Elterntrainings für Kinder der Sekundarstufe I. Zeitschrift für Entwicklungspsychologie und Pädagogische Psychologie, 36, 139 - 146. https://doi.org/10.1026/0049-8637.36.3.139

Bryan, T., Burstein, K. \& Bryan, J. (2001) Students with learning disabilities: Homework problems and promising practices. Educational Psychologist, 36, 167-180. https://doi.org/10. 1207/S15326985EP3603_3

Cote, L. R. \& Azar, S. T. (1997). Child age, parent and child gender, and domain differences in parents' attributions and responses to children's outcomes. Sex Roles, 36, 23 -50. https://doi.org/ 10.1007/BF02766237
Dilling, H., Mombour, W. \& Schmidt, M. H. (2015). Internationale Klassifikation psychischer Störungen: ICD-10 Kapitel V (F) Klinisch-diagnostische Leitlinien. Göttingen: Hogrefe.

Dumont, H., Trautwein, U., Lüdtke, O., Neumann, M., Niggli, A. \& Schnyder, I. (2012). Does parental homework involvement mediate the relationship between family background and educational outcome? Contemporary Educational Psychology, 37, 55-69. https://doi.org/10.1016/j.cedpsych.2011.09.004

Enlund, E., Aunola, K. \& Nurmi, J.-E. (2015). Stability in parents' causal attributions for their children's academic performance: A nine-year follow-up. Merrill-Palmer Quarterly, 61, 509-536. https://www.jstor.org/stable/10.13110/merrpalmquar1982.61. 4.0509

Esmaeeli, Z., Kyle, F. E. \& Lundetræ, K. (2019). Contribution of family risk, emergent literacy and environmental protective factors in children's reading difficulties at the end of second-grade. Reading \& Writing, 32, 2375 - 2399. https://doi.org/10.1007/ s11145-019-09948-5

Esser, G., Wyschkon, A. \& Schmidt, H. M. (2002). Was wird aus Achtjährigen mit einer Lese- und Rechtschreibstörung. Ergebnisse im Alter von 25 Jahren. Zeitschrift für Klinische Psychologie und Psychotherapie, 31, 235 -242. https://doi.org/10.1026/ 1616-3443.31.4.235

Fritzler, N. J. \& Wild, E. (2019). Bedingungen und Wirkung des elterlichen Engagements bei Schülerinnen und Schülern mit einer Lese-Rechtschreibstörung. Zeitschrift für Pädagogische Psychologie, 33, 71-84. https://doi.org/10.1024/1010-0652/ a000235

Gerber, J. \& Wild, E. (2009). Mit wem wird zu Hause wie gelernt? Die Hausaufgabenpraxis im Fach Deutsch. Unterrichtswissenschaft, 3, 213-229.

Levin, I., Levy-Shiff, R., Appelbaum-Peled, T., Katz, I., Komar, M. \& Meiran, N. (1997). Antecedents and consequences of maternal involvement in children's homework: A longitudinal analysis. Journal of Applied Developmental Psychology, 18, 207-227. https://doi.org/10.1016/S0193-3973(97)90036-8

Lonnemann, J, Linkersdörfer, J., Hasselhorn, M. \& Lindberg, S. (2011). Neurokognitive Korrelate der Dyskalkulie. Kindheit und Entwicklung, 20, 13-20. https://doi.org/10.1026/0942-5403/ a000036

Multhauf, B. \& Buschmann, A. (2014). Elternpartizipation in der Therapie lese-rechtschreib-schwacher Kinder. Ergebnisse einer Fragebogenerhebung. Zeitschrift für Kinder-und Jugendpsychiatrie und Psychotherapie, 42, 233 - 241. https://doi.org/ 10.1024/1422-4917/a000296

Natale, K., Aunola, K., Nurmi, J. E., Poikkeus, A. M., Lyytinen, P. \& Lyytinen, H. (2008). Mothers' causal attributions concerning the reading achievement of their children with and without familial risk for dyslexia. Journal of Learning Disabilities, 41, 274- 285. https://doi.org/10.1177/0022219408316094

Ng, F. F.-Y., Kenney-Benson, G. A. \& Poerantz, E. M. (2004). Children's achievement moderates the effects of mothers' use of control and autonomy support. Child Development, 75, 764780. https://www.jstor.org/stable/3696592

Núňez, J. C., Suárez, N., Rosário, P., Vallejo, G., Valle, A. \& Epstein, J. L. (2015). Relationships between perceived parental involvement in homework, student homework behaviors, and academic achievement. Metacognition \& Learning, 10, 375- 406.

Otto, B. (2007). SELVES - Schüler-, Eltern- und Lehrertrainings zur Vermittlung effektiver Selbstregulation. Berlin: Logos Verlag.

Pomerantz, E. M., Moormann, E. A. \& Litwack, S. D. (2007). The how, whom, and why of parents' involvement in children's academic lives: More is not always better. Review of Educational Research, 77, 373-410. https://doi.org/10.3102/00346543030 5567 
Roth, A. (2010). Entwicklung einer Skala zur Erfassung elterlicher Verhaltensintentionen in Lern- und Hausaufgabensituationen Bezüge zum akademischen Selbstkonzept bei einer Stichprobe lese-rechtschreibschwacher Schüler. Unveröffentlichte Diplomarbeit, Technische Universität Darmstadt.

Rytkönen, K., Aunola, K. \& Nurmi, J. E. (2005). Parents' causal attributions concerning their children's school achievement: A longitudinal study. Merrill-Palmer Quarterly, 51, 494-522. https://www.jstor.org/stable/23096099

Schulte-Körne, G., Deimel, W., Jungermann, M. \& Remschmidt, H. (2003). Nachuntersuchung einer Stichprobe von leserechtschreibgestörten Kindern im Erwachsenenalter. Zeitschrift für Kinder- und Jugendpsychiatrie und Psychotherapie, 31, $267-$ 276. https://doi.org/10.1024/1422-4917.31.4.267

Schulte-Körne, G., Warnke, A. \& Remschmidt, H. (2006). Zur Genetik der Lese-Rechtschreibschwäche. Zeitschrift für Kinderund Jugendpsychiatrie und Psychotherapie, 34, 435-444. https://doi.org/10.1024/1422-4917.34.6.435

Silinskas, G., Kiuru, N., Aunola, K., Lerkkanen, M.-K. \& Nurmi, J.-E. (2015). The developmental dynamics of children's academic performance and mothers' homework-related affect and practices. Developmental Psychology, 51, 419-433. https://doi.org/ 10.1037/a0038908

Silinskas, G., Niemi, P., Lerkkanen, M.-K. \& Nurmi, J.-E. (2012). Children's poor academic performance evokes parental homework assistance-but does it help? International Journal of Behavioral Development, 37, 44-56. https://doi.org/10.1177/ 0165025412456146
Weiner, B. (2014). The attribution approach to emotion and motivation: History, hypotheses, home runs, headaches/heartaches. Emotion Review, 6, 353-361. https://doi.org/10.1177/ 1754073914534502

\section{Danksagung}

Wir danken Dipl.-Psych. Anne Roth für die Erstellung des Elternfragebogens im Rahmen des Kosmos-Projektes.

\section{Förderung}

Die Daten wurden im Projekt „Kosmos - Kognitive und sozioemotionale Entwicklung von Kindern mit Lernstörung" erhoben, das am Frankfurter IDeA-Zentrum im Rahmen der Hessischen Landes-Offensive zur Entwicklung wissenschaftlich-ökonomischer Exzellenz (LOEWE) gefördert wurde.

Open Access-Veröffentlichung ermöglicht durch das DIPF | Leibniz-Institut für Bildungsforschung, Frankfurt am Main.

\section{Dr. Janin Brandenburg}

DIPF | Leibniz-Institut für Bildungsforschung

und Bildungsinformation

Rostocker Straße 6

60323 Frankfurt am Main

brandenburg@dipf.de 